\title{
Transcription factor RUNX2 regulates epithelial-mesenchymal transition and progression in renal cell carcinomas
}

\author{
BITIAN LIU ${ }^{1 *}$, JUNLONG LIU $^{2 *}$, HONGYUAN YU ${ }^{2}, \mathrm{CHANGMING} \mathrm{WANG}^{2}$ and CHUIZE KONG ${ }^{2}$ \\ ${ }^{1}$ Department of Urology, Shengjing Hospital of China Medical University, Shenyang, Liaoning 110004; \\ ${ }^{2}$ Department of Urology, The First Hospital of China Medical University, Shenyang, Liaoning 110001, P.R. China
}

Received July 3, 2019; Accepted November 25, 2019

DOI: 10.3892/or.2019.7428

\begin{abstract}
Renal cell carcinoma (RCC) is difficult to cure once it progresses and metastasizes. Runt-related transcription factor 2 (RUNX2) is associated with the development or progression of various cancers, but its role in RCC remains unclear. The expression of RUNX2 is not only aberrantly increased in ccRCC, but also is increased with increasing tumor stage and pathological grade. The prognosis of patients with tumors expressing RUNX2, which was revealed to be highly expressed in survival analysis, was significantly worse. Gene set enrichment analysis revealed that the RUNX2-mediated epithelial-mesenchymal transition (EMT) pathway promoted tumor progression. In vitro, knockdown of RUNX2 inhibited the proliferation, migration, and invasion of RCC, with related proteins in the EMT pathway exhibiting corresponding changes. RUNX2 regulated EMT in $\mathrm{RCC}$ to promote tumor progression.
\end{abstract}

\section{Introduction}

Renal cell carcinoma (RCC) represents the 6th most frequently diagnosed cancer in men and is 10th in women, accounting for 5 and $3 \%$ of all oncological diagnoses, respectively. According to the most updated data provided by the World Health Organization, there are more than 140,000 RCC-associated deaths yearly, with RCC ranking as the 13th most common cause of cancer-related deaths worldwide (1). The disease encompasses $>10$ histological and molecular subtypes, with clear cell RCC (ccRCC) accounting for approximately $75 \%$ of these subtypes and most cancer-related deaths (2). After recurrence or metastasis, ccRCC is largely incurable, and no specific marker can effectively predict or treat advanced cases.

Correspondence to: Professor Chuize Kong, Department of Urology, The First Hospital of China Medical University, 155 Nanjing North Street, Shenyang, Liaoning 110001, P.R. China E-mail: czkong_cmu@163.com

*Contributed equally

Key words: renal cell carcinoma, RUNX2, epithelial mesenchymal transition
Runt-related transcription factor 2 (RUNX2) belongs to the RUNX family of metazoan transcription factors, which serve as the major regulators of development with roles in proliferation, differentiation, apoptosis, and cell lineage specification. RUNX2, located on chromosome $6 \mathrm{p} 21$, is involved in bone formation and plays an important role in bone development (3). The RUNX2 protein plays a vital role in the pathogenesis of some cancers (4). Amplification of RUNX2 was revealed in osteosarcoma and has been proposed as an early event in its development (5). RUNX2 is also highly expressed in metastatic prostate cancer cells and is involved in promoting prostate cancer cell survival and bone tissue invasion (6). In breast cancer, RUNX2 can promote tumor invasion and metastasis, particularly during bone metastasis (7).

The role of RUNX2 in ccRCC has not been reported. Through bioinformatics and experimental verification, it was revealed that RUNX2 plays an important role in the progression of RCC. Combined with data from The Cancer Genome Atlas (TCGA) and Gene Expression Omnibus (GEO), the pathways and mechanisms involving RUNX2 were identified and evaluated. The functions of and pathways mediated by RUNX2 were demonstrated via experiments by silencing of RUNX2. These results may reveal the role of RUNX2 in promoting cancer progression in ccRCC.

\section{Materials and methods}

Data downloading and preprocessing. The RNA-sequencing (RNA-seq) results and corresponding clinical data from 611 tissues and 530 cases of ccRCC samples were obtained from TCGA database (https://portal.gdc.cancer.gov). These data were current as of May 7, 2019. RNA-seq results of 72 normal samples and 539 cancer samples were combined into a matrix file using a merge script in Perl language (http://www.perl.org/). Next, the Ensembl database (http://asia.ensembl.org/index. html) was used to convert gene names from the Ensembl IDs to a matrix of gene symbols. The data was normalized using the $\mathrm{R}$ package edge $\mathrm{R}$ and genes with expression levels of greater than one were retained. In the GEO database, GSE36133 (containing the expression profiles of $20 \mathrm{RCC}$ cell lines determined by array) was selected and downloaded.

Clinical significance of RUNX2. Using TCGA data downloaded in the previous step, the information was grouped 
according to sample type, tumor stage, and pathological grade to compare the expression of RUNX2. In the comparison process, 3 samples without stage information and 6 samples without grade information were excluded. Four cases lacked relevant follow-up information and were excluded, and the remaining 526 cases were divided into high and low groups according to the RUNX2 expression level for survival analysis by the log-rank test.

Gene set enrichment analysis (GSEA). Both tumor samples from TCGA and data from 20 RCC cell lines were divided into two groups according to the median expression of RUNX2. GSEA (http://software.broadinstitute.org/gsea/index.jsp) was used to identify the potential functions correlated with RUNX2 by assessing whether a series of a priori-defined biological processes was enriched in the gene rank derived from whole genes between the two groups. In the molecular signature database v6.2, hallmark gene sets of curated gene sets were used. Terms with Inormalized enrichment scorel $>1$, nominal $\mathrm{P}$-value $<0.05$, and false discovery rate of q-value $<0.25$ were identified.

Human samples. ccRCC and corresponding noncancerous tissues were obtained from patients at the Department of Urology at the First Hospital of China Medical University, between December 2016 and January 2018. There was a total of 40 patients, 28 males and 12 females, aged $41-78$ years, with mRNA samples from 14 patients (10 males, 4 females, age range 45-74 years) and protein samples from 26 other patients (18 males, 6 females, age range 41-78 years). The protocols used in the study were approved by the Hospital's Protection of Human Subjects Committee and written informed consent was obtained from all patients. The collected tissue samples were stored at $-80^{\circ} \mathrm{C}$ prior to use.

Cell culture and reagents. Cells from the human RCC cell lines 769-P, 786-O and OS-RC-2 were cultured in RPMI-1640 medium (HyClone; GE Healthcare Life Sciences), ACHN cells were cultured in minimal essential medium with Earle's balanced salts (HyClone; GE Healthcare Life Sciences), and Caki-1 cells were cultured in McCoy's 5A medium (Gibco; Thermo Fisher Scientific, Inc.). HK-2 cells (normal cortex/proximal tubule cells) were cultured in Dulbecco's modified Eagle's medium/F12 (HyClone; GE Healthcare Life Sciences). All culture media were supplemented with $10 \%$ fetal bovine serum. All cell lines were obtained from the Type Culture Collection of the Chinese Academy of Sciences (Shanghai, China) and cultured under humidified air containing $5 \% \mathrm{CO}_{2}$ at $37^{\circ} \mathrm{C}$. When the cells exceeded $80 \%$ confluence, they were washed with $1 \mathrm{X}$ phosphate-buffered saline (PBS) and trypsinized at $37^{\circ} \mathrm{C}$ for a specified amount of time for cell passage cultivation.

$R N A$ preparation and reverse transcription-quantitative polymerase chain reaction (RT-qPCR). Total RNA was extracted using ice-cold TRIzol reagent (Invitrogen; Thermo Fisher Scientific, Inc.) following the manufacturer's protocol. The concentration of total RNA was measured using Thermo Fisher Scientific NanoDrop ND-100. Total RNA was subjected to reverse transcription using the SYBR PrimeScript RT-PCR kit (Perfect Real-Time) (Takara Bio, Inc.). Real-time PCR was carried out using a Thermal Cycler Dice ${ }^{\mathrm{TM}}$ Real-Time system TP800 (Takara Bio, Inc.). The primer sequences designed for RUNX2 and $\beta$-actin were as follows ( $\left.5^{\prime}-3^{\prime}\right)$ : RUNX2 forward, GCGCATTCCTCAT CCCAGTA and reverse, GGCTCAGGT AGGAGGGGTAA; and $\beta$-actin forward, CATGTACGTTGC TATCCAGGC and reverse, CTCCTTAATGTCACGCAC GAT. The mRNA expression of the target gene was analyzed using the $2^{-\Delta \Delta \mathrm{Cq}}$ method (8).

Western blot analysis. Whole-cell lysates were extracted in radioimmunoprecipitation assay buffer containing $1 \mathrm{mM}$ phenylmethylsulfonyl fluoride (Beyotime Institute of Biotechnology), protease and phosphatase inhibitors. The concentration of protein samples was measured using a bicinchoninic acid assay (Beyotime Institute of Biotechnology). Equal amounts of protein $(30 \mu \mathrm{g} / \mathrm{lane})$ were separated by $10 \%$ SDS-PAGE (140 V) and the resolved proteins were transferred $(350 \mathrm{~mA})$ to polyvinylidene fluoride membranes $(0.2 \mu \mathrm{m})$ using a Mini-Trans-Blot apparatus (Bio-Rad Laboratories, Inc.). The membranes were blocked with $5 \%$ non-fat milk at $37^{\circ} \mathrm{C}$ for $1 \mathrm{~h}$, incubated with the primary antibody at $4^{\circ} \mathrm{C}$ overnight, and then incubated for $1 \mathrm{~h}$ with the appropriate secondary antibody. Proteins were finally detected with the EasySee ${ }^{\circledR}$ Western Blot kit (Beijing Transgen Biotech Co., Ltd.). The immunoblots were quantified using ImageJ software (version 1.51; National Institutes of Health). The optical density values from the immunoblots for samples and cells were normalized to the density values acquired for $\beta$-actin and GAPDH, respectively. The monoclonal primary antibodies were: Anti-E-cadherin (dilution 1:5,000; product code ab40772), anti-N-cadherin (dilution 1:5,000; product code ab76011) and anti-vimentin (dilution 1:1,000; product code ab92547; all from Abcam), anti-p44/42MAPK (anti-ERK; dilution 1:1,000; product no. 4695), anti-phospho-p44/42MAPK (anti-p-ERK; dilution 1:2,000; product no. 4370), anti-RUNX2 (dilution 1:1,000; product no. 12556) and anti-GAPDH (dilution 1:1,000; D16H11; all from Cell Signaling Technology, Inc., and anti- $\beta$-actin (dilution 1:5,000; cat. no. 66009-1-Ig; ProteinTech Group, Inc.).

Small interfering RNA (siRNA) transfection. The 786-O and Caki-1 cells (cultured to $70 \%$ confluence) were seeded into 6-well plates at a density of $4 \times 10^{5}$ cells/well and transfected with two effective RUNX2 siRNAs (JTS Scientific). siRNA transfection was performed using Lipofectamine 3000 (Invitrogen; Thermo Fisher Scientific, Inc.) according to the product's protocol. After $6 \mathrm{~h}$, the medium was replaced, and the cells were cultured for another 24 or $48 \mathrm{~h}$ for further assays. The siRNA and negative control (NC) siRNA sequences used were as follows (5'-3'): siRNA-1 sense, CACGCUAUUAAA UCCAAAUTT and antisense, AUUUGGAUUUAAUAGCGU GTT; siRNA-2 sense, CAAGUCCUUUUAA UCCACATT and antisense, UGUGGAUUAAA AGGACUUGGT; and NC sense, UUCUCCGAACGUGUCACGUTT and antisense, ACGUGACACGUUCGGAGAATT.

Transwell assays. Cell migration was measured using Transwell chambers with $8-\mu \mathrm{m}$ pores in 24 -well tissue culture plates (Corning Costar, Corning, Inc.). For cell invasion assays, Transwell chambers coated with Matrigel (BD Biosciences) 
were inserted into a 24 -well plate. After transfection of siRNA for $24 \mathrm{~h}$, the cells were re-suspended in medium without fetal bovine serum and $0.2 \mathrm{ml}$ of cell suspension $\left(2 \times 10^{4}\right.$ cells/well for cell migration; $4 \times 10^{4}$ cells/well for cell invasion) was seeded into the top chamber, whereas the lower chamber of each well was filled with $0.6 \mathrm{ml}$ of medium containing $10 \%$ fetal bovine serum to act as a chemoattractant. After 12-24 h of incubation at $37^{\circ} \mathrm{C}$, the cells that remained on the upper side of the filter were removed using cotton swabs and those that had migrated to the lower side were fixed with $4 \%$ paraformaldehyde for $10 \mathrm{~min}$, and stained with $1.0 \%$ crystal violet for $10 \mathrm{~min}$ at room temperature. Images were captured with an EVOS ${ }^{\mathrm{TM}}$ XL Core Imaging system (Invitrogen; Thermo Fisher Scientific, Inc.) and cells were counted using ImageJ software (version 1.51; National Institutes of Health).

5-Ethynyl-2'-deoxyuridine (EdU). 786-O and Caki-1 cells were seeded into 24 -well plates at a density of $1 \times 10^{4}$ cells/well and cultured for $24 \mathrm{~h}$. The solution of an EdU Kit (BeyoClick ${ }^{\mathrm{TM}}$ EDU Cell Proliferation Kit with Alexa Fluor 488; Beyotime Institute of Biotechnology) was diluted 1:1,000 in cell medium. The cells were incubated with the EdU solution for $2 \mathrm{~h}$ at $37^{\circ} \mathrm{C}$. Following fixation in $4 \%$ paraformaldehyde for $15 \mathrm{~min}$ at room temperature and treatment with $0.3 \%$ Triton-X for $15 \mathrm{~min}$ at room temperature, the cells were incubated for $30 \mathrm{~min}$ with Click reaction cocktail in a dark room at room temperature. Prior to observation under a fluorescence microscope, nuclei were stained with Hoechst 33342.

Interaction of proteins. STRING (https://www.string-db. org) version 11.0 was used with multiple protein modules for protein interaction network analysis.

Gene co-expression analysis. The co-expression relationships between RUNX2 and related genes were determined based on gene expression levels to determine the strength of the relationships at the transcriptional level. The $\mathrm{R}$ corrplot package was used to calculate the Pearson correlation between genes.

Statistical analysis. All data were expressed as the mean \pm standard deviation (SD) and represented as the average of at least three experiments with each experiment performed in triplicate. Statistical significance was determined using the Student's t-test (two-tailed) for 2 groups, one-way analysis of variance or (and) Tukey's test for more than 2 groups. Correlations between the expression levels of different genes were evaluated by Spearman correlation analysis. In the bar graphs *,**, ***, ****, and $\mathrm{ns}$ indicate $\mathrm{P}<0.05, \mathrm{P}<0.01, \mathrm{P}<0.001$, $\mathrm{P}<0.0001$, and not significant, respectively. A P-value $<0.05$ was considered to indicate a statistically significant difference.

\section{Results}

Expression and survival analysis. Box-and-Whisker plots revealed RUNX2 expression levels (based on sample type) in normal and tumor samples (Fig. 1A). The expression was higher in all individual cancer stages than in adjacent normal tissues, and stage 4 exhibited the highest RUNX2 expression (Fig. 1B). Comparisons of the grades revealed that RUNX2 expression had a more evident increasing trend (Fig. 1C).
Survival analysis revealed that the RUNX2 gene significantly affected prognosis (Fig. 1D). The aforementioned results indicated that RUNX2 is an important gene in ccRCC and its mRNA expression is upregulated during tumor development.

GSEA. To identify the potential function of RUNX2, GSEA was conducted to search for pathways enriched in the higher-RUNX2-expressed samples. The functions and pathways potentially enriched by RUNX2 were separately analyzed in the previously downloaded TCGA cancer samples and GEO cell line data. The purity of the cancer samples affected our analysis results, however the cell lines were not influenced by purity, but had a small total sample size. Therefore, the screening conditions for the cell lines were relaxed and the results obtained to determine the functions and pathways potentially affected by RUNX2 were combined (Fig. 1E). The IL6/JAK/STAT3 signaling axis in cancer drives the proliferation, survival, invasiveness, and metastasis of tumor cells while strongly suppressing the antitumor immune response (9). In the IL2/STAT5 signaling pathway, STAT5 was revealed to play a critical role in the function and development of regulatory $\mathrm{T}$ cells (Tregs), and consistently activated STAT5 was associated with the suppression of antitumor immunity and increased proliferation, invasion, and survival of tumor cells (10). Epithelial-mesenchymal transition (EMT) is associated with tumor stemness, metastasis, and therapy resistance (11). The three pathways and mechanisms aforementioned were correlated with RUNX2 and promoted tumor progression. Overexpression of RUNX2 was related to the inflammatory response and allograft rejection (Fig. 1F).

RUNX2 mRNA and protein levels in ccRCC and RCC cell lines. The clinical tissue specimens collected from our hospital were analyzed to verify the results of bioinformatics analyses and it was confirmed that RUNX2 mRNA expression was significantly upregulated in tumor tissues compared to normal kidney tissues in 14 paired samples (Fig. 2A). To examine RUNX2 protein expression in clinical ccRCC specimens, another 26 pairs of cancer tissues and normal tissues were assessed. Western blot results indicated a significant increase of RUNX2 protein expression in tumor tissues compared to adjacent normal tissues (Fig. 2B and C). These data indicated that RUNX2 was highly expressed in kidney tumors and may be a reliable target for further investigation. Next, RUNX2 mRNA and protein levels were assessed in RCC cell lines and the cell line with the highest RUNX2 expression (780-O) and that with the lowest expression (Caki-1) were selected for subsequent experiments (Fig. 2D and E). Notably, the EMT pathway-associated proteins in 786-O were significantly different from those in Caki-1, consistent with the high activation of the RUNX2-mediated EMT mechanism predicted by GSEA (Fig. 2F).

Migration and invasion. Transwell assays were performed to explore the effect of RUNX2 on the migration and invasion of RCC cells. Decreasing RUNX2 expression with siRNA in 786-O and Caki-1 cells inhibited cell migration and invasion (Fig. 3A and B). Combined with upregulation of E-cadherin and downregulation of $\mathrm{N}$-cadherin, vimentin and phosphorylated extracellular signal-regulated kinase ( $\mathrm{p}$-ERK) proteins after transfection, RUNX2 was revealed to be associated 

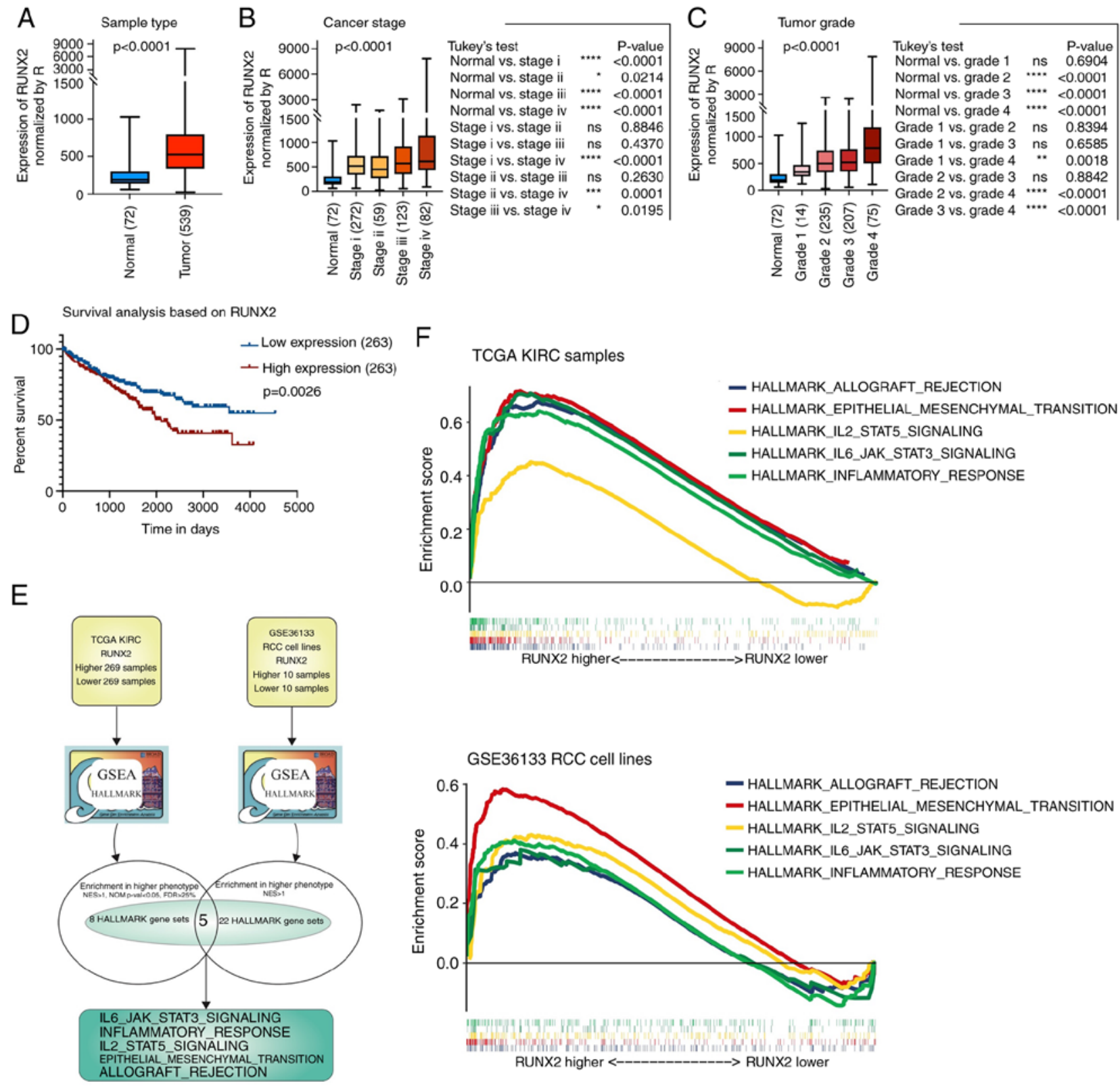

Figure 1. Clinical significance of RUNX2 in ccRCC and RUNX2-correlated enrichment gene sets analyzed by GSEA. Expression of RUNX2 based on (A) sample types, (B) cancer stages and (C) pathological grade. (D) Survival analysis based on expression of RUNX2. (E) Acquisition process of RUNX2-related enrichment gene sets. (F) Enrichment score of five enrichment gene sets in TCGA tumor samples and GSE36133 RCC cell lines. RUNX2, runt-related transcription factor 2; ccRCC, clear cell renal cell carcinoma; GSEA, gene set enrichment analysis. ${ }^{*} \mathrm{P}<0.05,{ }^{* * *} \mathrm{P}<0.01,{ }^{* * * *} \mathrm{P}<0.001,{ }^{* * * * *} \mathrm{P}<0.0001$, ns, not significant.

with the EMT pathway in renal clear cell carcinoma, which is consistent with the previous results of GSEA. The gray value of the western blot of RUNX2 in Caki-1 (Fig. 3A) was significantly higher than that of RUNX2 (Fig. 2E) in the previous cell line comparison. This was because the sample load was increased by 3 -fold and the exposure time was extended to 6 min (exposure time in Fig. 3E is 2 min).

Proliferation. EdU assays revealed that cell proliferation was decreased when RUNX2 was silenced, indicating that RUNX2 enhanced tumor growth in vitro (Fig. 3C).

Identification of core-related genes. Overexpression of RUNX2 is related to ccRCC progression. Tumor progression is a complex biological process involving multiple cellular pathways and mechanisms. RUNX2 has been revealed to be involved in the EMT pathway, the IL6/JAK/STAT3 signaling pathway, and the IL2/STAT5 signaling pathway (screened by GSEA), wherein the relationship with the core enrichment genes may be direct or indirect. IL6/JAK/STAT signaling has been revealed to promote EMT (12). These enriched pathways and mechanisms have similar biological functions and thus the genes shared among them may be critical. The core enrichment genes of the EMT, IL6/JAK/STAT3 signaling, and IL2/STAT5 signaling pathways were analyzed to identify the genes revealing significant relationships with RUNX2, and then the genes were combined from TCGA and cell lines for subsequent analysis (Fig. 4A).

Protein-protein interaction and co-expression of core-related genes. In STRING, the proteins from the 19 core genes mostly 

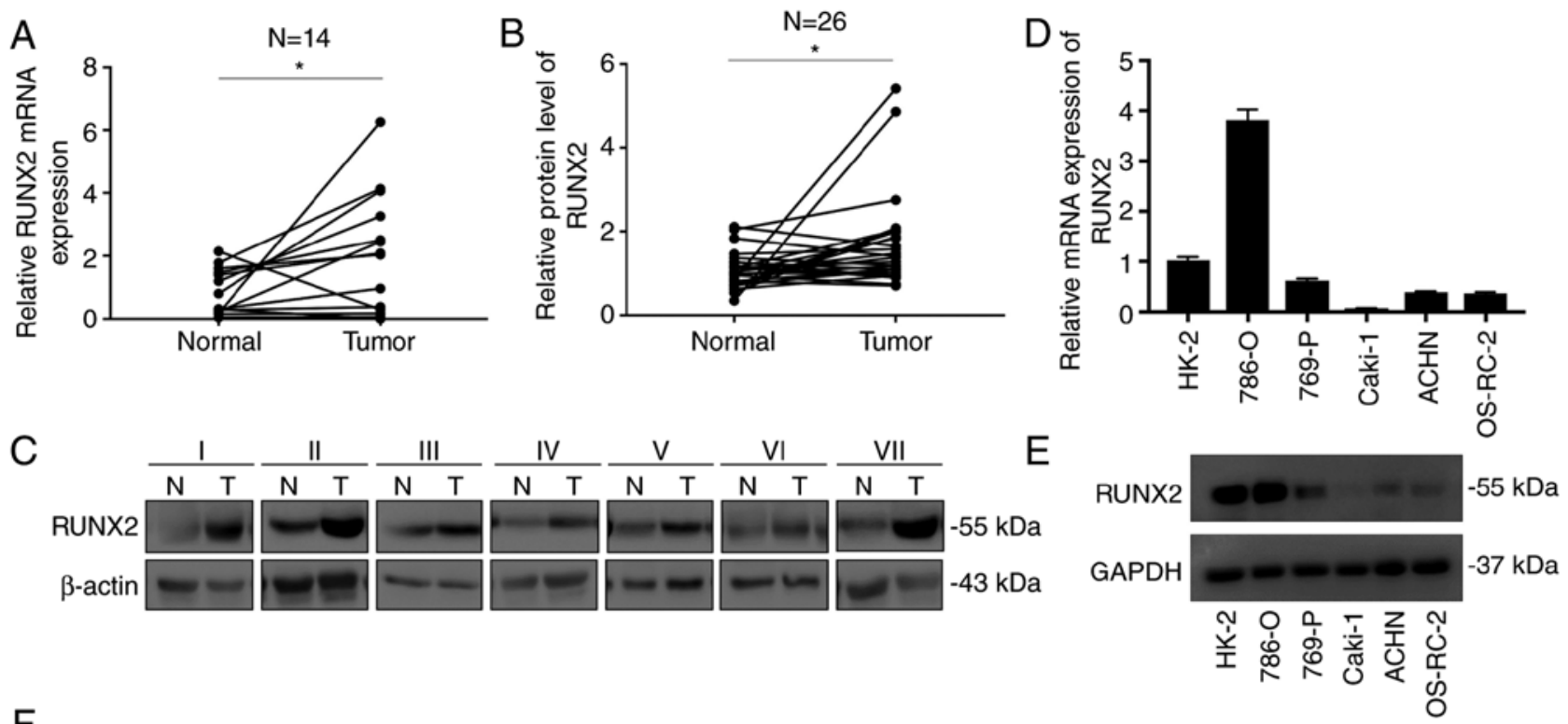

$\mathrm{F}$
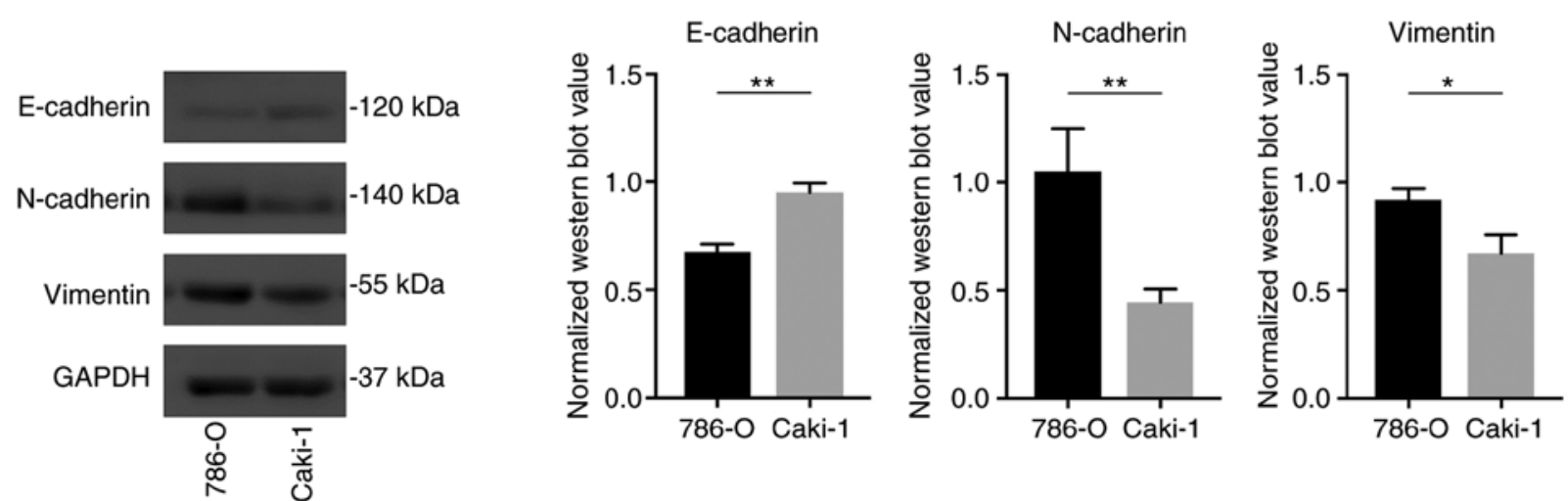

Figure 2. RUNX2 is upregulated in ccRCC tissues. (A) RUNX2 expression levels were detected in 14 pairs of ccRCC and adjacent normal tissue samples by qPCR. (B) RUNX2 protein levels were detected in 26 pairs of ccRCC and adjacent normal tissue samples by western blot analysis and results were quantified (C) Western blot analysis revealed that the RUNX2 protein was overexpressed in cancer tissues compared to non-tumor tissues. (D) qPCR analysis revealed that RUNX2 mRNA was observed in all six cell lines. (E) Western blot analysis revealed that the RUNX2 protein was expressed in all six cell lines. (F) EMT markers regarding migration capabilities exhibited different expression patterns in low-RUNX2 Caki-1 and high-RUNX2 786-O cells. RUNX2, runt-related transcription factor 2; ccRCC, clear cell renal cell carcinoma; qPCR, quantitative PCR; EMT, epithelial-mesenchymal transition. ${ }^{*} \mathrm{P}<0.05$, ${ }^{* *} \mathrm{P}<0.01$.

revealed protein interactions (Fig. 4B). Among them, IL6 exhibited the most extensive effect, whereas RUNX2 exhibited protein interactions with 5 other proteins. According to TCGA ccRCC tumor data, RUNX2 was also positively correlated with most core-related gene transcriptional levels normalized by $\log 2$ (Fig. 4C). CSF2 was ruled out because its expression was too low.

\section{Discussion}

RUNX2 was first demonstrated to promote cancer progression in RCC. The RUNX2-related EMT pathway determined by GSEA was related to tumor invasion and metastasis. The results of the Transwell and EDU experiments confirmed the role of RUNX2 in promoting tumor progression, and the EMT pathway-related proteins were subsequently altered after silencing of RUNX2. The genes shared by the GSEA screening pathways may be essential for RUNX2 to promote tumor progression.

When searching for enriched pathways, data from RCC cell lines were also included to more accurately identify cellular pathways potentially involving RUNX2. RCC cell lines can be considered as cancer cells extracted from the tumor environment, where interference from other cells is excluded. Based on the results of GSEA, RUNX2 may mediate EMT. 786-O cells with the high expression of RUNX2 were compared to Caki-1 cells with low expression of RUNX2, and their EMT-related proteins exhibited the same trend. Silencing of RUNX2 could reduce the phosphorylation level of ERK, which is a key coordinator of EMT (13).

Based on the GSEA results, it was revealed that overexpression of RUNX2 was associated with IL6/JAK/STAT3 signaling, IL2/STAT5 signaling, and EMT and that overexpression may be directly or indirectly related to these pathways or mechanisms in promoting tumor progression. However, RUNX2 upregulation also positively regulated the inflammatory response and allograft rejection, indicating that the body is consistently undergoing an immune response during tumor progression. However, the process of immune clearance was strongly inhibited. STAT3 is frequently overactivated in tumor-infiltrating immune cells, negatively regulating neutrophils, natural killer (NK) cells, effector T cells, and dendritic cells (DCs), suggesting 

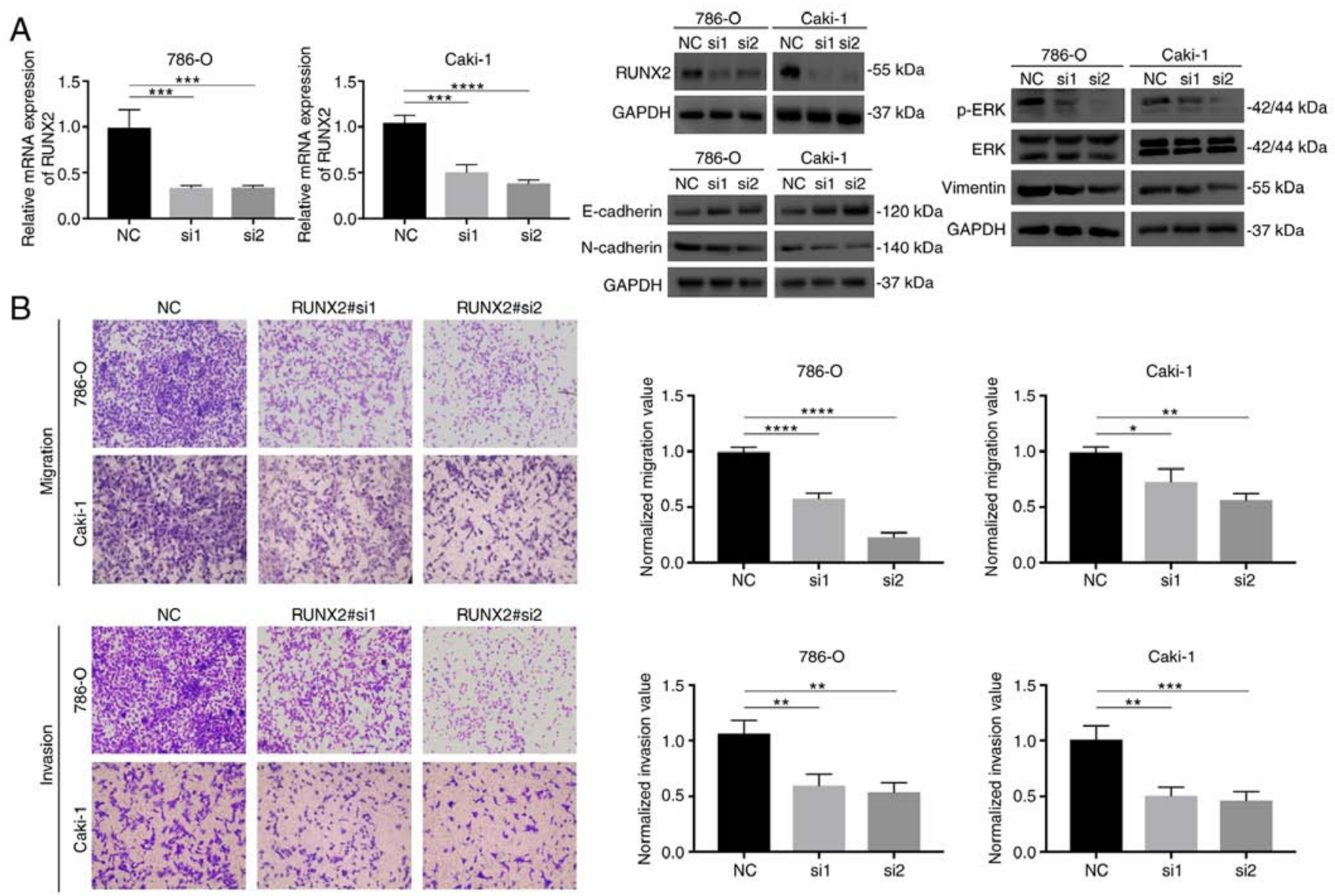

C
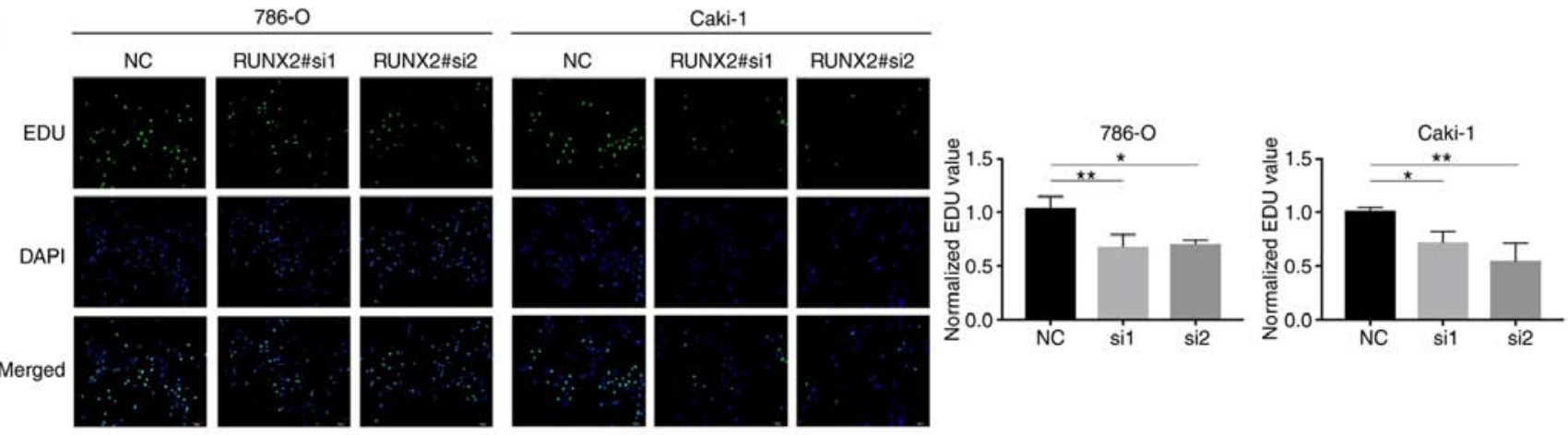

Figure 3. RUNX2 promotes cell migration, invasion, and proliferation. (A) qPCR and western blot analysis revealed that RUNX2 was downregulated by siRNA, and that EMT-related proteins subsequently changed. (B) Migration and invasion of 786-O and Caki-1 after RUNX2 silencing. (C) Proliferation of 786-O and Caki-1 after RUNX2 silencing. Data were analyzed by Tukey's test and presented as the mean \pm standard deviation (SD). RUNX2, runt-related transcription factor 2; qPCR, quantitative PCR; EMT, epithelial-mesenchymal transition. ${ }^{*} \mathrm{P}<0.05,{ }^{* * *} \mathrm{P}<0.01,{ }^{* * *} \mathrm{P}<0.001,{ }^{* * * * *} \mathrm{P}<0.0001$.

that activation of STAT3 results in downregulation of antitumor immunity (9). STAT5 plays a critical role in the function and development of Tregs, and consistently activated STAT5 is associated with the suppression of antitumor immunity (10). EMT also promotes immune escape (14), and thus activation of the aforementioned pathway mechanisms may lead not only to tumor progression, but also to resistance and evasion of the immune system. Moreover, once the inflammatory response fails to control the tumor, it is typically exploited by the tumor to promote its own growth and progression to metastasis (15).

When searching for possible RUNX2-related genes, correlation analysis was not performed for all genes. Moreover, the expression level was first subjected to $\log 2$ transformation to reduce the scale of expression over different orders of magnitude and render the data more stable. We first searched for core genes in the enrichment pathway through GSEA, and then conducted correlation analysis to identify potentially associated genes. This approach is more biologically meaningful.

Both CD44 and transforming growth factor beta 1 (TGF $\beta 1$ ) have a relatively strong relationship with RUNX2 as revealed through protein interactions and co-expression. The protein encoded by CD44 is a cell-surface glycoprotein involved in cell-cell interaction, cell adhesion, and migration. CD44, the only gene shared by all enrichment pathways, may have protein interactions and be co-expressed with RUNX2. In prostate 
A
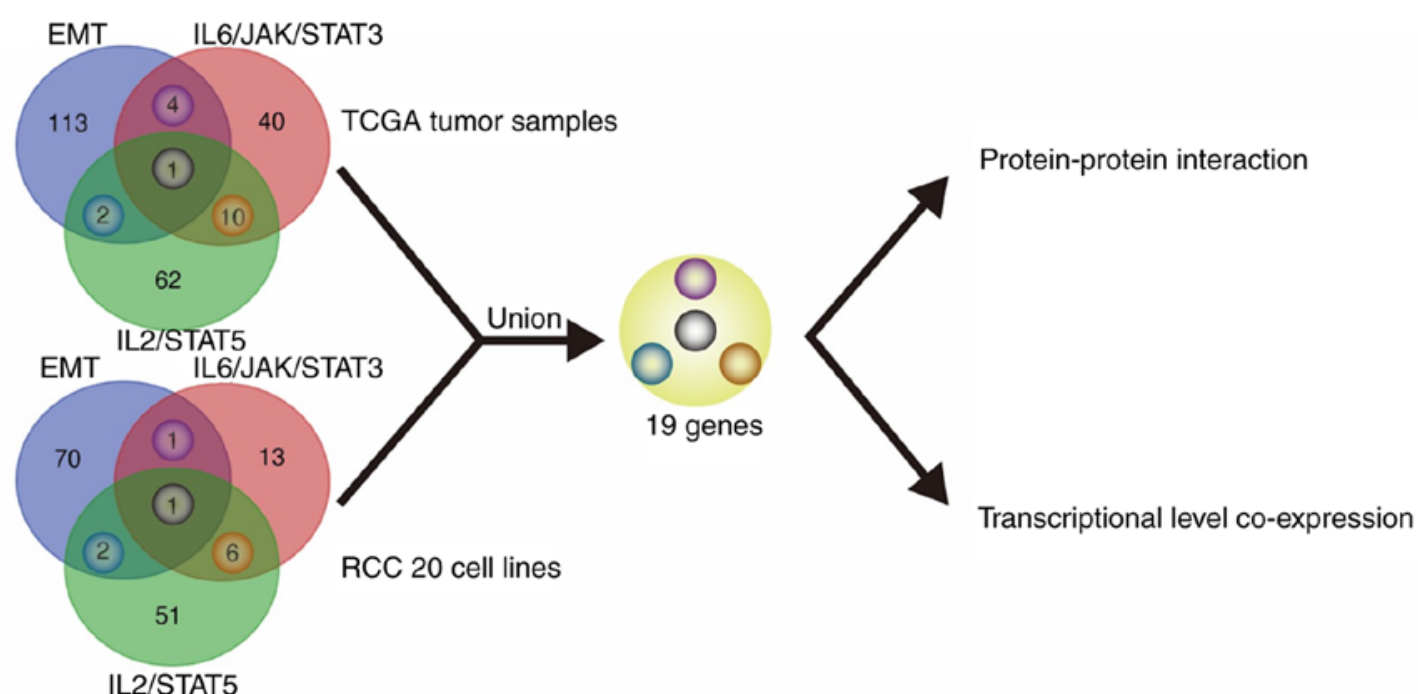

B

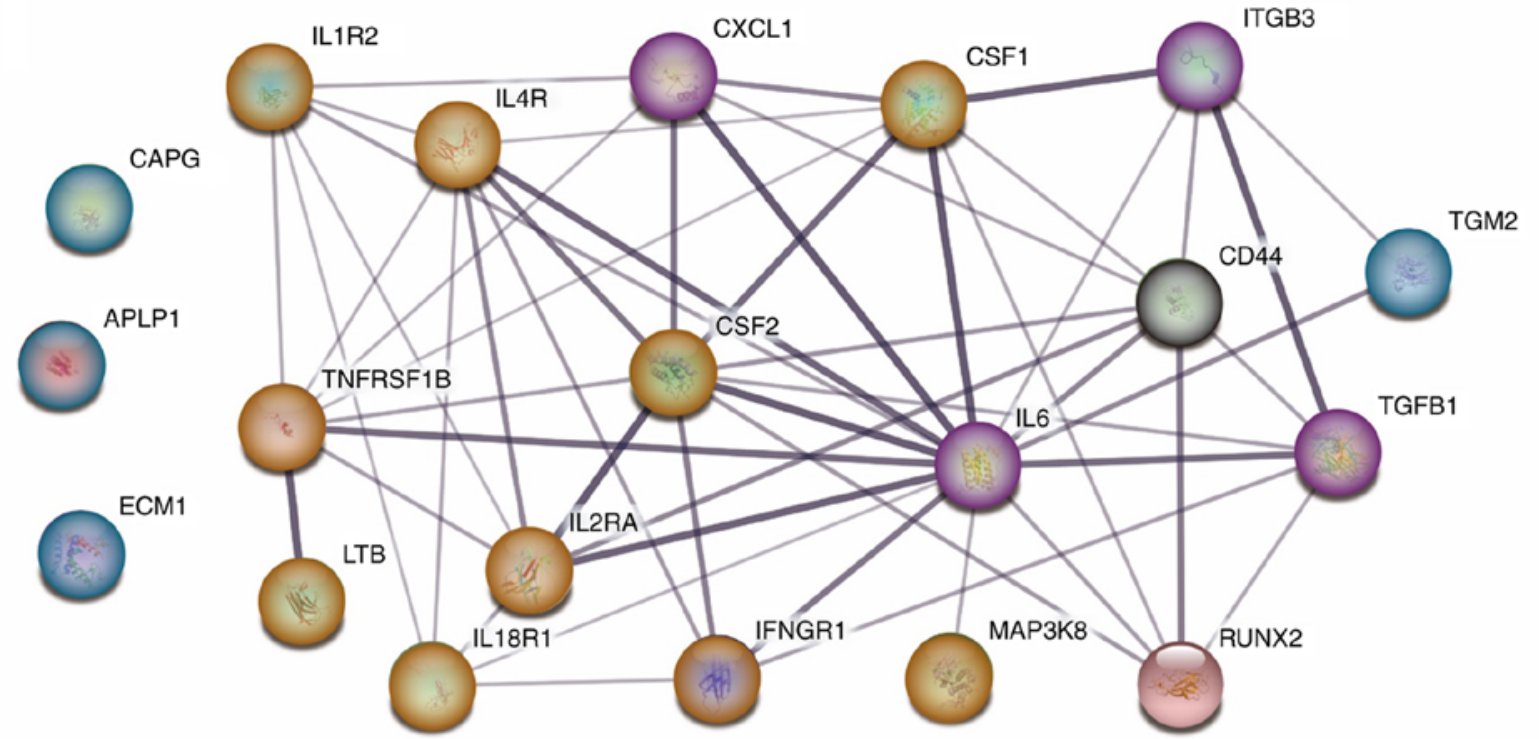

C
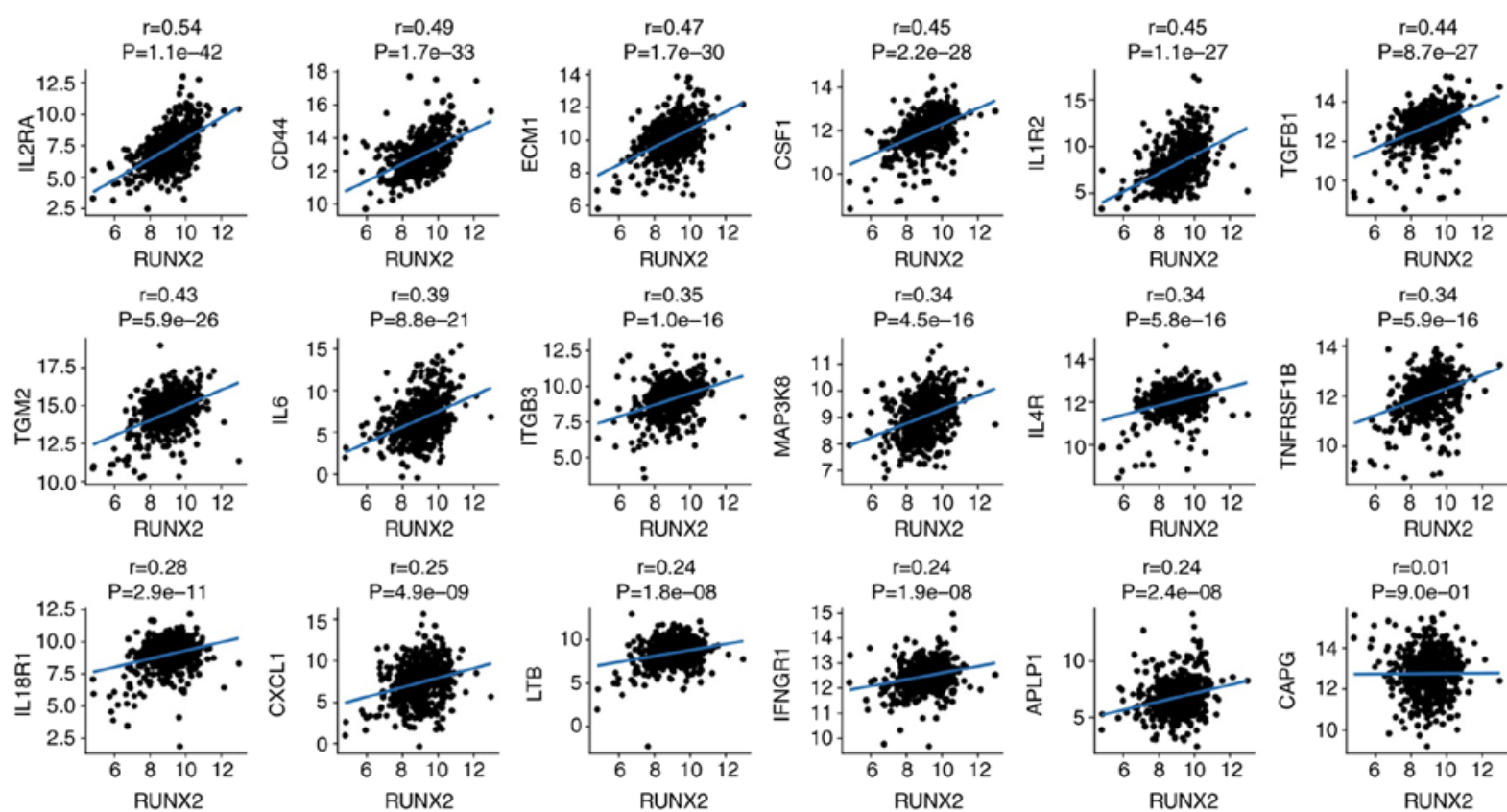

Figure 4. (A) Acquisition process for potential RUNX2 functionally-related genes. (B) Protein-protein interaction between RUNX2 and 19 functionally-related genes. (C) Transcription level correlation between RUNX2 and 19 functionally-related genes. RUNX2, runt-related transcription factor 2. 
cancer PC3 cells, knockdown of CD44 reduced RUNX2 expression at the mRNA and protein levels, thereby reducing RUNX2-mediated signaling (16). In addition, CD44 expression may play an important role in the progression of RCC (17). RUNX2 was revealed to be a target of TGF $\beta 1$ in $\mathrm{C} 2 \mathrm{C} 12$ pluripotent mesenchymal precursor cells and was involved in regulating the TGF- $\beta$ pathway (18). In RCC, TGF $\beta 1$ was revealed to be significantly associated with tumor stage T3-4, Fuhrman III and IV, and tumor size $>4 \mathrm{~cm}$ (19).

RUNX2-related IL6/JAK/STAT3 signaling, IL2/STAT5 signaling, and core-related proteins were not experimentally verified. This will be evaluated in our future studies.

In general, RUNX2 plays a role in promoting tumor progression in RCC. RUNX2 drives the EMT process to promote RCC migration and invasion. Additional studies are required to determine the mechanism by which RUNX2 promotes RCC progression. RUNX2 may be a useful therapeutic target for RCC.

\section{Acknowledgements}

Not applicable.

\section{Funding}

The present study was supported by the National Natural Science Fund (grant no. 81672525), the Liaoning Natural Science Fund (grant no. 201602830), and China Medical University's 2017 discipline promotion program (grant no. 2017XK08).

\section{Availability of data and materials}

The datasets used and/or analyzed during the current study are available from the corresponding author on reasonable request. Publicly available datasets can be found here: https://portal. gdc.cancer.gov.

\section{Authors' contributions}

BL designed the study and drafted the manuscript. JL and BL performed the experiments. BL collected, analyzed, and interpreted the data. $\mathrm{HY}$ and $\mathrm{CW}$ collected tissue samples of the patients with RCC and contributed to revision of the manuscript and figures. CK acquired funding, established the urology laboratory, provided the required equipment and instruments, contributed to the critical reading of the manuscript. All authors read and approved the manuscript and agree to be accountable for all aspects of the research in ensuring that the accuracy or integrity of any part of the work are appropriately investigated and resolved.

\section{Ethics approval and consent to participate}

The present study was approved by the Ethics Committee on Human Research of the First Affiliated Hospital of China Medical University (Shenyang, China), and written informed consent was obtained from all patients.

\section{Patient consent for publication}

Consent for publication was obtained from all participants.

\section{Competing interests}

The authors declare no competing financial interests. The funding agency did not participate in the design of the study and collection, analysis, and interpretation of data or in writing of the manuscript.

\section{References}

1. Capitanio U, Bensalah K, Bex A, Boorjian SA, Bray F, Coleman J, Gore JL, Sun M, Wood C and Russo P: Epidemiology of renal cell carcinoma. Eur Urol 75: 74-84, 2019.

2. Hsieh JJ, Purdue MP, Signoretti S, Swanton C, Albiges L, Schmidinger M, Heng DY, Larkin J and Ficarra V: Renal cell carcinoma. Nat Rev Dis Primers 3: 17009, 2017.

3. Liu Z, Yao X, Yan G, Xu Y, Yan J, Zou W and Wang G: Mediator MED23 cooperates with RUNX2 to drive osteoblast differentiation and bone development. Nat Commun 7: 11149, 2016.

4. Ito Y, Bae SC and Chuang LS: The RUNX family: Developmental regulators in cancer. Nat Rev Cancer 15: 81-95, 2015.

5. Martin JW, Zielenska M, Stein GS, van Wijnen AJ and Squire JA: The role of RUNX2 in osteosarcoma oncogenesis. Sarcoma 2011: 282745, 2011

6. Akech J, Wixted JJ, Bedard K, van der Deen M, Hussain S, Guise TA, van Wijnen AJ, Stein JL, Languino LR, et al: Runx2 association with progression of prostate cancer in patients: Mechanisms mediating bone osteolysis and osteoblastic metastatic lesions. Oncogene 29: 811-821, 2010.

7. Li XQ, Du X, Li DM, Kong PZ, Sun Y, Liu PF, Wang QS and Feng YM: ITGBL1 Is a Runx2 transcriptional target and promotes breast cancer bone metastasis by activating the TGF $\beta$ signaling pathway. Cancer Res 75: 3302-3313, 2015.

8. Livak KJ and Schmittgen TD: Analysis of relative gene expression data using real-time quantitative PCR and the 2(-Delta Delta C(T)) method. Methods 25: 402-408, 2001.

9. Johnson DE, O'Keefe RA and Grandis JR: Targeting the IL-6/JAK/STAT3 signalling axis in cancer. Nat Rev Clin Oncol 15: 234-248, 2018.

10. Rani A and Murphy JJ: STAT5 in cancer and immunity. J Interferon Cytokine Res 36: 226-237, 2016.

11. Pastushenko I, Brisebarre A, Sifrim A, Fioramonti M, Revenco T, Boumahdi S, Van Keymeulen A, Brown D, Moers V, Lemaire $\mathrm{S}$, et al: Identification of the tumour transition states occurring during EMT. Nature 556: 463-468, 2018.

12. Xiao J, Gong Y, Chen Y, Yu D, Wang X, Zhang X, Dou Y, Liu D, Cheng G, Lu S, et al: IL-6 promotes epithelial-to-mesenchymal transition of human peritoneal mesothelial cells possibly through the JAK2/STAT3 signaling pathway. Am J Physiol Renal Physiol 313: F310-F318, 2017.

13. Shin S, Buel GR, Nagiec MJ, Han MJ, Roux PP, Blenis J and Yoon SO: ERK2 regulates epithelial-to-mesenchymal plasticity through DOCK10-dependent Rac1/FoxO1 activation. Proc Natl Acad Sci USA 116: 2967-2976, 2019.

14. Nan X, Wang J, Liu HN, Wong STC and Zhao H: Epithelial-mesenchymal plasticity in organotropism metastasis and tumor immune escape. J Clin Med 8: E747, 2019.

15. Dominguez C, David JM and Palena C: Epithelial-mesenchymal transition and inflammation at the site of the primary tumor. Semin Cancer Biol 47: 177-184, 2017.

16. Gupta A, Cao W and Chellaiah MA: Integrin $\alpha v \beta 3$ and CD 44 pathways in metastatic prostate cancer cells support osteoclastogenesis via a Runx2/Smad 5/receptor activator of NF- $\kappa$ B ligand signaling axis. Mol Cancer 11: 66, 2012.

17. Lee YM, Kim JM, Lee HJ, Seong IO and Kim KH: Immunohistochemical expression of CD44, matrix metalloproteinase 2 and matrix metalloproteinase 9 in renal cell carcinomas. Urol Oncol 37: 742-748, 2019.

18. Lee KS, Kim HJ, Li QL, Chi XZ, Ueta C, Komori T, Wozney JM, Kim EG, Choi JY, Ryoo HM and Bae SC: Runx 2 is a common target of transforming growth factor betal and bone morphogenetic protein 2, and cooperation between Runx2 and Smad5 induces osteoblast-specific gene expression in the pluripotent mesenchymal precursor cell line C2C12. Mol Cell Biol 20: 8783-8792, 2000.

19. Lebdai S, Verhoest G, Parikh H, Jacquet SF, Bensalah K, ChautardD, Rioux Leclercq N, Azzouzi AR and Bigot P: Identification and validation of TGFBI as a promising prognosis marker of clear cell renal cell carcinoma. Urol Oncol 33: 69.e11-e68, 2015. 